\title{
Fat Grafting for Hand Rejuvenation
}

\author{
Christopher J. Conlon, MD ${ }^{1}$ Amjed Abu-Ghname, MD ${ }^{1}$ Matthew J. Davis, BS ${ }^{1} \quad$ Kausar Ali, MD ${ }^{1}$ \\ Sebastian J. Winocour, MD, MSc, FACS ${ }^{1}$ Michael L. Eisemann, MD ${ }^{2}$ Julian Winocour, MD ${ }^{3}$
}

1 Division of Plastic Surgery, Michael E. DeBakey Department of
Surgery, Baylor College of Medicine, Houston, Texas
2 Department of Plastic and Reconstructive Surgery, Methodist
Hospital, Houston, Texas
${ }^{3}$ Department of Plastic Surgery, Vanderbilt University Medical
Center, Nashville, Tennessee

Semin Plast Surg 2020;34:47-52.

\author{
Address for correspondence Julian J. Winocour, MD, Department of \\ Plastic Surgery, Vanderbilt University Medical Center, D-4207 Medical \\ Center North, Nashville, TN 37232 \\ (e-mail: julian.winocour@vanderbilt.edu).
}

\begin{abstract}
Keywords

- fat grafting

- lipofilling

- autologous fat transfer

- hand rejuvenation

The hands are one of the most visible parts of the body, and prominent dorsal veins and extensor tendons are the most readily recognized signs of the aging process. Fat grafting has been demonstrated to be a safe and effective method of hand rejuvenation by restoration of subcutaneous fat. Despite some variability in the technical approach, fat grafting techniques are consistent in their use of low-pressure injection with standard cannula sizes, small aliquots of graft, and a total volume of graft greater than or equal to $15 \mathrm{~mL}$ per hand. While distribution of the fat is an area of debate and a topic of active research, published studies have shown high patient satisfaction rates, suggesting that perhaps the restoration of volume alone is paramount. In this article, we will review the applications of fat grafting to the hand, focusing primarily on its role in hand rejuvenation.
\end{abstract}

Fat grafting for the correction of concavities dates back over a century. ${ }^{1}$ Lipofilling of the hand was first introduced in $1988 .^{2}$ The technique involved deposition of a fat bolus in the proximal dorsum of the hand, with subsequent massaging to achieve contour. ${ }^{2}$ A flurry of innovative strategies followed. In 1989, lipofilling was combined with laser resurfacing. ${ }^{3}$ Hand rejuvenation with microlipoinjection for age-related cosmetic improvement was introduced in $1990 .{ }^{4}$ The first published patient series reached the literature in 1992, reporting excellent patient satisfaction (98.6\%). ${ }^{5}$ In 2002, Coleman published a landmark paper detailing a method for structural fat grafting. ${ }^{6}$ The technique of making multiple passes with depositions of small quantities produced more consistent results when contrasted with injecting a lump of fat and manipulating it throughout the hand. ${ }^{6}$

\section{The Aging Process of the Hand}

The hands are the most visible unclothed body part, apart from the face. Accordingly, they play an outsized role in the visible aging process. People are able to roughly determine the age of a person by examining the dorsum of the hands. ${ }^{7-9}$ The natural aging process of the hand leads to changes in the quality and texture of the skin and atrophy of the soft tissue and muscles, leading to an increased prominence and visibility of the dorsal veins and extensor tendons. ${ }^{10}$ In a survey study by Bains et al, the authors showed images of female hands of different ages to the general public. ${ }^{7}$ Digitally altered photographs with decreased prominence of veins were judged to show younger hands than in the original photograph, with a greater effect on perceived age than other cosmetic changes such as jewelry and nail polish. ${ }^{7}$

Aging of the hand is characterized by both soft tissue atrophy of the dermis, fascia, fat, and interosseous muscle and a decline in skin quality secondary to sun exposure (-Fig. 1). ${ }^{10}$ Soft tissue thickness of the dorsum of the hand decreases with age, averaging $3.12 \mathrm{~mm}$ in adolescence and $1.6 \mathrm{~mm}$ in patients older than 45 years. ${ }^{10}$ The soft tissue atrophy is metered by skin thickness decreasing from $1.2 \mathrm{~mm}$ at 25 years of age to $0.75 \mathrm{~mm}$ at 70 years of age, with concomitant declines in the quantity and quality of collagen and elastin in the dermis. ${ }^{11,12}$ Dorsal hand skin thickness is
Issue Theme Fat Grafting in Plastic Surgery; Guest Editor: Sebastian J. Winocour, MD, MSc, FACS
Copyright (c) 2020 by Thieme Medical Publishers, Inc., 333 Seventh Avenue, New York, NY 10001, USA. Tel: +1(212) 760-0888.
DOI https://doi.org/ 10.1055/s-0039-1700960. ISSN 1535-2188. 


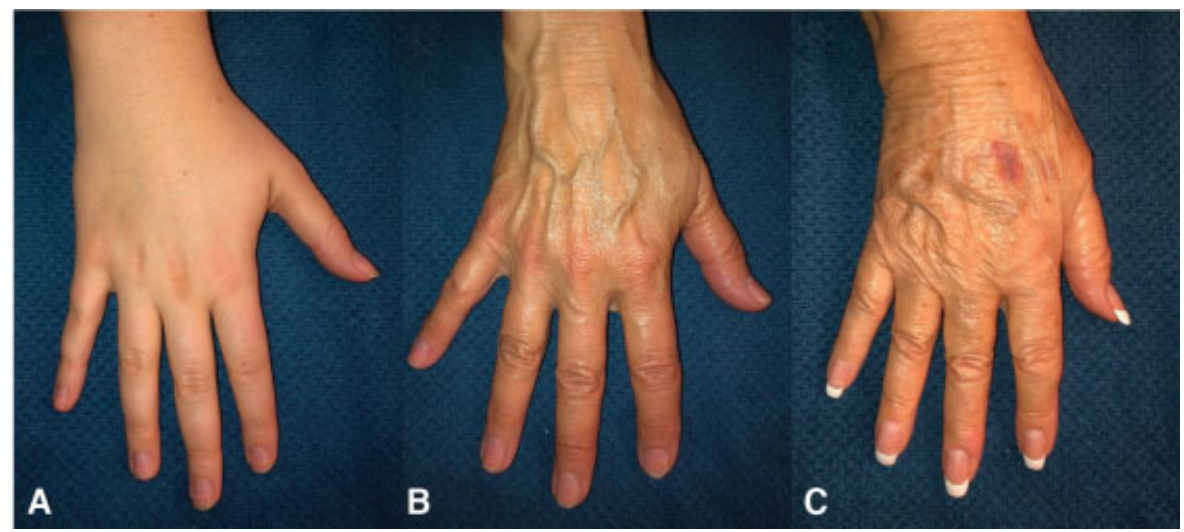

Fig. 1 (A-C) Aging of the hand. (A) Hand photograph at the age of 20 years. (B) Hand photograph at the age of 50 years. (C) Hand photograph at the age of 80 years.

influenced by gender, with a predilection for thicker skin in males $-0.05 \mathrm{~mm}$ at old age. ${ }^{11}$ Atrophy of the soft tissues creates the characteristic concavities between the metacarpals. ${ }^{10}$ In addition, characteristic lentigines, dyschromia, textural roughness, seborrheic, and/or actinic keratosis distinguish the aged hand from the youthful one. ${ }^{12}$ Hand aesthetics and skin quality are influenced by genetics, smoking and alcohol abuse, chronic sun exposure, overwork, chemical toxin exposure, and rheumatological disease. ${ }^{12,13}$

\section{Ideal Hand Proportions and Relevant Anatomy}

The hand has long been studied as a measure of attractiveness, and ideal, aesthetic hand proportions have been well described. Hand length is $11 \%$ of the total body length, $49 \%$ of which is made up by the long finger. Hand width is $45 \%$ of the hand length. The dominant hand is significantly larger, although the effect is diminished in left-handed individuals due to greater relative ambidexterity. ${ }^{14}$ Females have $25 \%$ less hand volume than males of the same height. ${ }^{13,15}$ These average proportions can serve as a critical point of reference when making cosmetic alterations to the hand.

Fat distribution in the dorsum of the hand can be divided into three laminae: superficial, intermediate, and deep (-Fig. 2)..$^{10,16,17}$ The laminae are separated by fascial planes. They are bounded proximally by the extensor retinaculum, where the fascial layers fuse. Veins cross into the hand superficial to the extensor retinaculum, whereas tendons run deep. The laminae are bounded distally by the metacarpophalangeal joints.

The superficial lamina begins less than $1 \mathrm{~mm}$ deep to the skin surface and contains no important structures. ${ }^{16,18}$ The fat distribution is uneven in this layer. The layer has 8 to 12

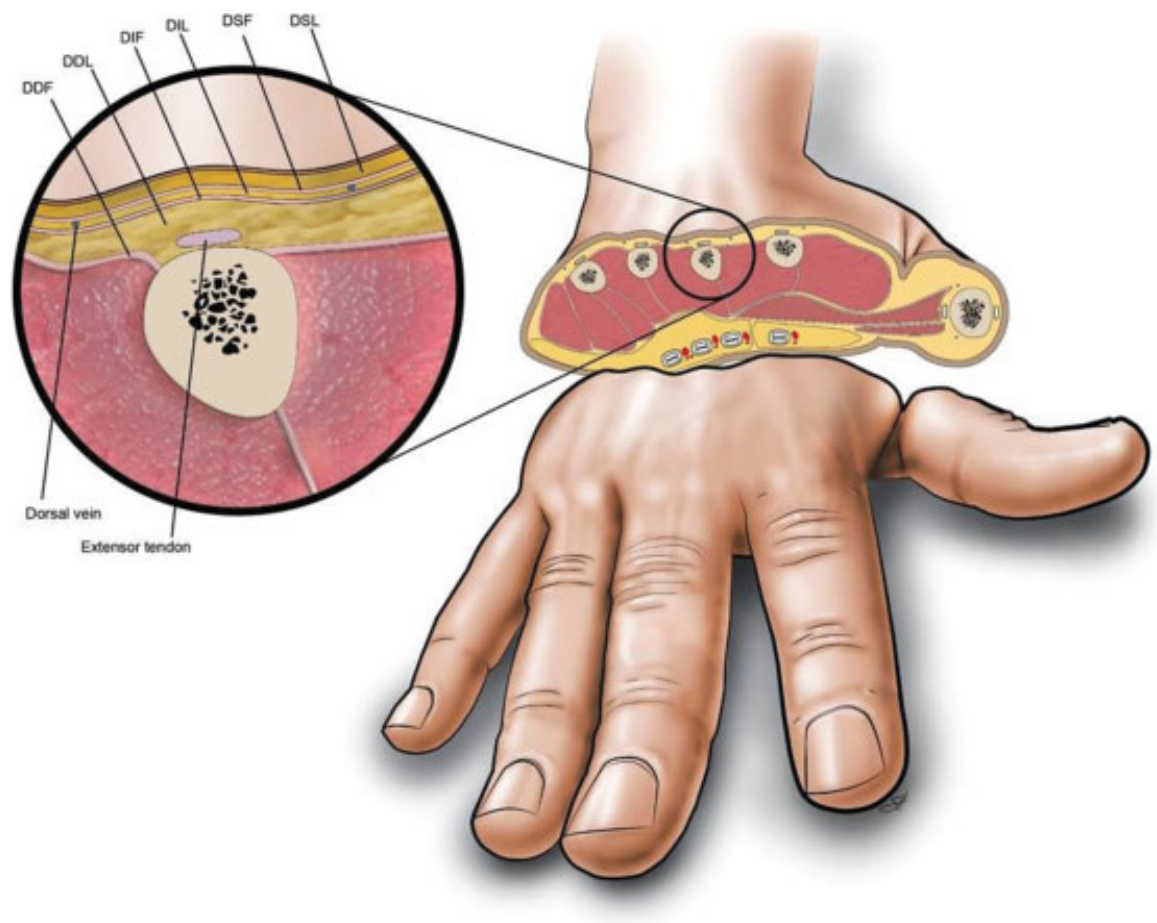

Fig. 2 Cross-section of hand transmetacarpals. (Reproduced with permission of Baylor College of Medicine.) 
spanning fascial septae, which contain small vessels. ${ }^{16,18}$ The intermediate lamina contains the veins and nerves of the dorsum of the hand. Dorsal veins have an average diameter of $1.27 \mathrm{~mm} .{ }^{19}$ The deep lamina comprises four compartments with intervening extensor tendons.

\section{Assessing the Hand}

Scientific analysis of the aesthetic of the hand begins with the Kretschmer classification according to body habitus. ${ }^{20}$ The aesthetic hand, associated with the ectomorphic body type, is characterized by long, slender hands and is considered the most desirable type. The rough, wide, and balanced hand-the athletic hand-is associated with the mesomorphic body type. The least aesthetically pleasing classification is the pyknic type: a hand with a short, wide dorsum and short, conical fingers associated with an endomorphic body type. Continued focus on the aesthetics of the hand yielded a remarkably robust characterization of the hand dimensions.

Zhou et al offered a subjective grading system for the soft tissue volumes in the hand. ${ }^{10} \mathrm{~A}$ grade of 0 is consistent with a natural and smooth hand contour. Hands with grade 1 exhibit mild atrophy with rhytides. Grade 2 soft tissue volume loss is consistent with moderate atrophy with exposed veins. Grade 3 soft tissue volume loss exhibits serious atrophy with exposed tendons. ${ }^{10}$ The grade of soft tissue deficiency helps to determine the necessary corrective action. Grade 1 volume loss can be managed with nonsurgical treatment, whereas grade 2 and grade 3 volume losses are best rectified with increasing degrees of surgical intervention. As an alternative to this qualitative scale, Zhou et al have also put forth a quantitative system of measuring soft tissue thickness with B-mode ultrasound. ${ }^{10}$

Other qualitative measures have been used in attempts to quantify patient's dorsal hand volume. The Merz Hand Grading Scale is a 5-point photonumeric scale developed specifically to assess improvement after hand rejuvenation intervention. ${ }^{21}$ The Global Aesthetic Improvement Scale is a 5 -point Likert scale that is nonspecific to the hand and quantifies change with intervention.

\section{Fat Grafting and Harvest}

The tenets of fat delivery to the hand are low pressure, low speed, and low volume through multiple tunnels and multiple planes. ${ }^{6,10,22}$ During delivery, attention must be focused on delivering the smallest aliquots of fat possible in an even manner in the desired planes at a low pressure. Specialized devices are under investigation to facilitate the safe and effective delivery. ${ }^{22}$

Access incisions for fat grafting vary between protocols. A proximal incision distal to the extensor retinaculum provides access to the superficial lamina and the proximal dorsum of the hand. ${ }^{10,23}$ An incision in the webspace between the first and second metacarpals accomplishes a similar goal. ${ }^{17}$ Incisions in the other webspaces provide good access to the deeper lamina and the distal portion of the dorsum of the hand with minimal scar burden. ${ }^{10,17}$ Some experts recom- mend a single incision between the third and fourth metacarpals to further decrease scar burden. ${ }^{17}$

There is controversy regarding which laminae are ideal for fat deposition. Widespread agreement exists regarding the safety and efficacy of injecting into the superficial lamina only. $^{10,17,22}$ The superficial lamina contains no important structures, and the fat graft covers the deeper vessels and tendons. The intermediate lamina contains the dorsal veins, and some experts recommend avoiding this lamina to avoid intravascular fat deposition. ${ }^{10}$ Others, however, argue in support of fat grafting to this lamina to maximize treatment efficacy without serious concerns about safety. ${ }^{17,22}$ Similarly, grafting the deep lamina is recommended by some experts to correct concavity between the metacarpal bones, whereas others do not believe it is necessary to fat graft that deep in the hand. ${ }^{10,17,22}$

Fat delivery by cannula is the preferred method. Cannulas sized between 14 and 17 gauge are standard. ${ }^{2,10,17,22-24}$ Cannulas larger than 14 gauge are associated with deposits susceptible to central necrosis. ${ }^{24}$ Cannulas smaller than 17 gauge have an outer diameter smaller than the diameter of the dorsal hand veins and carry a greater theoretical risk of intravascular fat deposition. ${ }^{19}$

Several surgeons have published their individual techniques for fat grafting to the hand and have recommended different amounts of fat to ideally be transferred. Early reports were modest in their recommendations of transfer. ${ }^{2,6}$ The most recent suggestions agree that 15 to $30 \mathrm{~mL}$ of fat is indicated for grafting the dorsum of each hand, depending on the technique used and the degree of correction desired. ${ }^{10,17,22,23}$ Some surgeons recommend an overcorrection in anticipation of graft loss, whereas others stress that apparent graft loss is in fact because of the prolonged resolution of edema and recommend against overcorrection. 6,10

There is a wide variability that exists in techniques described for fat harvest. However, there is little evidence at this time to support a significant difference between different donor sites, donor-site preparations, harvest techniques, fat harvesting cannula sizes, and centrifugation speeds. ${ }^{25}$ Nevertheless, many experts in the field have shared their techniques as guidance. Most practitioners use the classic adiposities of the flanks, the periumbilical areas, or the medial thighs as donor sites. The internal thigh has been proposed as the ideal site due to purported superior graft take and, anecdotally, less patient discomfort. ${ }^{17}$ Widespread adoption of the needle-syringe unit has been the standard practice since its introduction by Fournier in $2000 .^{2}$ A dry liposuction technique is recommended by some experts due to the relatively low volume of lipoaspirate required. ${ }^{17}$ Nevertheless, a complete review of fat harvest techniques and outcomes is beyond the scope of this study.

\section{Efficacy}

Fat grafting to the hand is an effective treatment for age-related volume loss and subsequent prominence of vasculature and tendons (-Fig. 3). As methods have become more refined, Fournier's claim that results persist beyond 4 to 5 years has held 


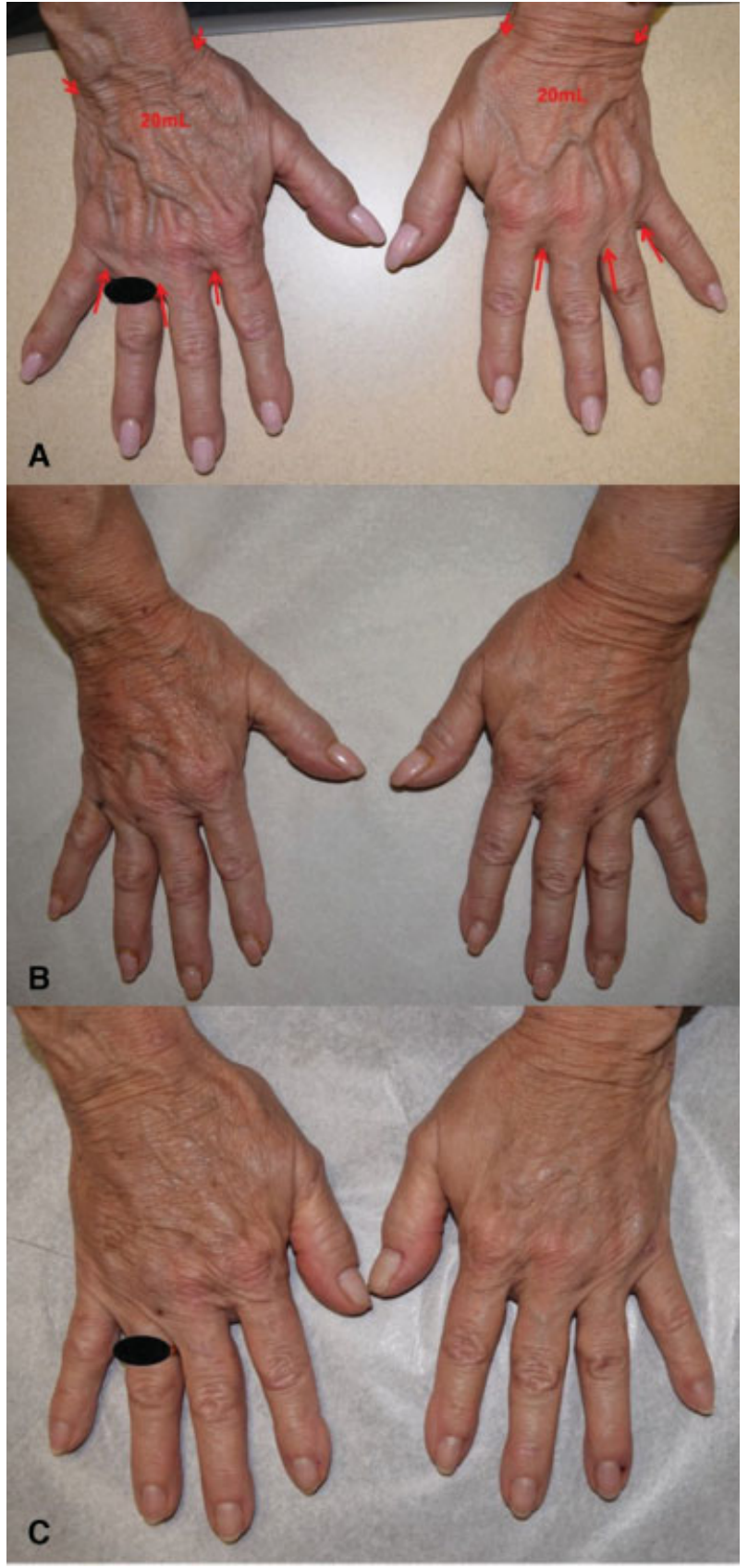

Fig. 3 Bilateral hand rejuvenation using fat grafting. (A) Preoperative hand photograph. (B) One day after fat grafting. (C) Three months after fat grafting.

true. ${ }^{2}$ Volume gain is seen in all patients, with fat retention rates comparable to fat grafting in other locations. ${ }^{10,26}$ Subjective measures are in concordance with the objective measures of change. Satisfaction ratings are consistently reported in the mid-1990s in published case series. ${ }^{10,22,23}$

\section{Complications}

Edema is the most commonly reported complication following fat grafting. Some practitioners have proposed that edema is part of the natural healing process after fat grafting to the hand and not considered a true complication since edema in the dorsum of the hand resolves spontaneously. ${ }^{10,22,23}$ Compression dressings applied over several days can help manage the immediate postoperative edema. ${ }^{6,17}$ Other spontaneously resolving complications include ecchymoses and paresthesias, which presumably occur due to damage to small vascular and neural structures, respectively. ${ }^{10,23}$ Infections in the hand represent a more significant degree of complication. Abscesses following hand rejuvenation have been described as isolated case reports. ${ }^{27,28}$ Antibiotic prophylaxis with a cephalosporin has been used to diminish risk. ${ }^{23}$

\section{Therapeutic Fat Grafting for Noncosmetic Pathologies}

Besides its use in aesthetic hand rejuvenation, fat grafting has been used to treat patients with Raynaud's phenomenon and Dupuytren's disease using similar techniques. ${ }^{29,30}$ Patients with Raynaud's phenomenon report reduced pain, fewer cold attacks, improved skin and soft tissue texture, fewer ulcerations, and subjectively improved function following 10 to $15 \mathrm{~mL}$ of fat grafting to the hand. The protocol used by Bank et al calls for the fat to be divided between the dorsal and volar webspaces, the snuffbox, and the superficial palmar arch. The mechanism of action is not clear, as no significant changes in blood flow were observed. ${ }^{29}$

Fat grafting has also been used to treat patients with Dupuytren's disease. In fact, when affected rays were treated with $10 \mathrm{~mL}$ of fat grafting, patients reported high levels of satisfaction (96\%). At early follow-up, contracture was significantly improved at the proximal interphalangeal joint and resolved at the metacarpophalangeal joint. ${ }^{31}$ Patients were able to resume occupational activities 9 days after the operation on average. ${ }^{32}$ However, while no difference in recurrence rates was observed at 1-year follow-up when compared with limited fasciectomy, an increased recurrence rate was observed at 5-year followup, which has raised some concerns about the utility of this procedure. $^{32,33}$

\section{Integration of Fat Grafting with Other Hand Rejuvenation Modalities}

Fat grafting is one option in the larger pantheon of hand rejuvenation techniques that restore tissue volume, which includes fillers, hyaluronic acid, and poly-l-lactic acid. Artificial fillers have been approved by the U.S. Food and Drug Administration (FDA) as safe and efficacious alternatives to fat grafting. These fillers differ from fat grafts in that they achieve a similar result in volume restoration without any risk of donor-site morbidity. However, their treatment length is temporary, with the longevity dependent on the product. Nevertheless, these fillers play an important role by providing an alternative for patients who do not want any donorsite morbidity.

Calcium hydroxyapatite (Radiesse, Merz Aesthetics) was approved by the FDA in $2015 .{ }^{34}$ A recommended quantity of 1.5 to $3 \mathrm{~mL}$ per dorsum is administered through incisions in 
the distal webspace. ${ }^{12,18}$ These fillers are effective for a reported 6 to 12 months. ${ }^{12}$

Hyaluronic acid (Restylane Lyft with Lidocaine, Galderma Laboratories) was approved by the FDA in $2018 .{ }^{35}$ A recommended quantity of $2 \mathrm{~mL}$ per dorsum, divided into four equal portions between the webspaces, is delivered into the superficial and intermediate laminae by injecting into tented skin. ${ }^{36}$ It also produces a 6- to 12 -month effect. ${ }^{35,36}$

Poly-l-lactic acid (Sculptra Aesthetic, Galderma Laboratories) is used off-label for hand rejuvenation. It is currently approved by the FDA only for lipoatrophy secondary to HIV antiretrovirals and nasolabial fold contour deficiencies. ${ }^{37}$ An administration of $2 \mathrm{~mL}$ per dorsum is delivered through a cannula to the undersurface of the dermis. ${ }^{38,39}$ It reportedly has an effect for 18 to 24 months, with a proposed long-term biostimulatory increase in collagen. ${ }^{38,40}$

Other hand rejuvenation techniques cannot restore volume but are effective in changing the aesthetic of the superficial dorsum. Excision of a dorsal ellipse from the wrist-with the long axis perpendicular to the long axis of the arm-creates a smoother and more youthful dorsum but leaves a visible scar. ${ }^{8}$ Chemical peels with trichloroacetic acid or phenol are associated with dermal thickening and lightening skin pigmentation. ${ }^{9,41}$ Laser therapy, sclerotherapy, pulsed light therapy, and topical tretinoin can resurface the dorsal skin to produce a more youthful aesthetic. ${ }^{8}$

\section{Conclusion}

The hands are one of the most visible body parts-second only to the face-and prominent dorsal veins and extensor tendons are the most readily recognized signs of the aging process due to soft tissue atrophy. Fat grafting has been shown to be a safe and effective method of restoring a more youthful appearance to the hand by restoring dorsal hand volume. The restoration of subcutaneous fat covers visible tendons and veins, decreases skin laxity, and promotes local dermal regeneration. Deeper fat placement corrects concavities that naturally occur between the metacarpals during the aging process. As edema resolves over the first few months after the procedure, long-term, stable results are achieved.

The recommended fat grafting techniques, while variable in some respects, are consistent in their use of low-pressure injection with standard cannula sizes, small aliquots of graft, and a total volume of graft greater than or equal to $15 \mathrm{~mL}$ per hand. Where to distribute the fat is an area of disagreement and topic of active research; however, all published studies have shown exceptional satisfaction rates, suggesting that perhaps the restoration of volume alone is paramount.

\section{Conflicts of Interest}

None of the authors has a financial interest in any of the products, devices, or drugs mentioned in this manuscript.

\section{Acknowledgments}

Special thanks to Scott Holmes, CMI, for his graphic assistance in the preparation of this manuscript.

\section{References}

1 Neuber F. Fettransplantation. Bericht über die Verhandlungen der deutschen Gesellscaft für Chirurgie. Zentralbl Chir 1893;22:66

2 Fournier PF. Who should do syringe liposculpturing? J Dermatol Surg Oncol 1988;14(10):1055-1056

3 Abergel RP, David LM. Aging hands: a technique of hand rejuvenation by laser resurfacing and autologous fat transfer. J Dermatol Surg Oncol 1989;15(07):725-728

4 Abrams HL, Lauber JS. Hand rejuvenation. The state of the art. Dermatol Clin 1990;8(03):553-561

5 Aboudib Júnior JH, de Castro CC, Gradel J. Hand rejuvenescence by fat filling. Ann Plast Surg 1992;28(06):559-564

6 Coleman SR. Hand rejuvenation with structural fat grafting. Plast Reconstr Surg 2002;110(07):1731-1744, discussion 1745-1747

7 Bains RD, Thorpe H, Southern S. Hand aging: patients' opinions. Plast Reconstr Surg 2006;117(07):2212-2218

8 Wendt JR. Distal, dorsal superior extremity plasty. Plast Reconstr Surg 2000;106(01):210-213

9 Butterwick KJ. Rejuvenation of the aging hand. Dermatol Clin 2005;23(03):515-527, vii

10 Zhou J, Xie Y, Wang WJ, et al. Hand rejuvenation by targeted volume restoration of the dorsal fat compartments. Aesthet Surg J 2017;38(01):92-100

11 Brodar V. Observations of skin thickness and subcutaneous tissue in man. Z Morphol Anthropol 1960;50:386

12 Rivkin AZ. Volume correction in the aging hand: role of dermal fillers. Clin Cosmet Investig Dermatol 2016;9:225-232

13 Jakubietz RG, Jakubietz MG, Kloss D, Gruenert JG. Defining the basic aesthetics of the hand. Aesthetic Plast Surg 2005;29(06): 546-551

14 Von Lanz T, Wachsmuth W. Praktische anatomie. Vol. 1/3. 1st ed. Berlin, Germany: Springer; 1959

15 Burger M. Die hand on Kranken. 1st ed. Munich, Germany: Lehmanns Verlag; 1956

16 Bidic SM, Hatef DA, Rohrich RJ. Dorsal hand anatomy relevant to volumetric rejuvenation. Plast Reconstr Surg 2010;126(01):163-168

17 Villanueva NL, Hill SM, Small KH, Rohrich RJ. Technical refinements in autologous hand rejuvenation. Plast Reconstr Surg 2015; 136(06):1175-1179

18 Frank K, Koban K, Targosinski S, et al. The anatomy behind adverse events in hand volumizing procedures: retrospective evaluations of 11 years of experience. Plast Reconstr Surg 2018;141(05): $650 \mathrm{e}-662 \mathrm{e}$

19 Kiray A, Ergür I, Tayefi H, Bağriyanik HA, Bacakoğlu AK. Anatomical evaluation of the superficial veins of the upper extremity as graft donor source in microvascular reconstructions: a cadaveric study. Acta Orthop Traumatol Turc 2013;47(06):405-410

20 Kretschmer E. Physique and Character. 1st ed. Berlin, Germany: Springer; 1931

21 Carruthers A, Carruthers J, Hardas B, et al. A validated hand grading scale. Dermatol Surg 2008;34(Suppl 2):S179-S183

22 Yun-Nan L, Shu-Hung H, Tsung-Ying L, et al. Micro-autologous fat transplantation for rejuvenation of the dorsal surface of the aging hand. J Plast Reconstr Aesthet Surg 2018;71(04):573-584

23 Fantozzi F. Hand rejuvenation with fat grafting: a 12-year singlesurgeon experience. Eur J Plast Surg 2017;40(05):457-464

24 James IB, Bourne DA, DiBernardo G, et al. The architecture of fat grafting II: impact of cannula diameter. Plast Reconstr Surg 2018; 142(05):1219-1225

25 Strong AL, Cederna PS, Rubin JP, Coleman SR, Levi B. The current state of fat grafting: a review of harvesting, processing, and injection techniques. Plast Reconstr Surg 2015;136(04):897-912

26 Giunta RE, Eder M, Machens HG, Müller DF, Kovacs L. Structural fat grafting for rejuvenation of the dorsum of the hand [in German]. Handchir Mikrochir Plast Chir 2010;42(02):143-147

27 Galea LA, Nicklin S. Mycobacterium abscessus infection complicating hand rejuvenation with structural fat grafting. J Plast Reconstr Aesthet Surg 2009;62(02):e15-e16 
28 Vara AD, Miki RA, Alfonso DT, Cardoso R. Hand fat grafting complicated by abscess: a case of a bilateral hand abscess from bilateral hand fat grafting. Hand (N Y) 2013;8(03):348-351

29 Bank J, Fuller SM, Henry GI, Zachary LS. Fat grafting to the hand in patients with Raynaud phenomenon: a novel therapeutic modality. Plast Reconstr Surg 2014;133(05):1109-1118

30 Khouri RK Jr, Khouri RK. Current clinical applications of fat grafting. Plast Reconstr Surg 2017;140(03):466e-486e

31 Hovius SE, Kan HJ, Smit X, Selles RW, Cardoso E, Khouri RK. Extensive percutaneous aponeurotomy and lipografting: a new treatment for Dupuytren disease. Plast Reconstr Surg 2011;128 (01):221-228

32 Hovius SE, Kan HJ, Verhoekx JS, Khouri RK. Percutaneous aponeurotomy and lipofilling (PALF): a regenerative approach to Dupuytren contracture. Clin Plast Surg 2015;42(03):375-381, ix

33 Selles RW, Zhou C, Kan HJ, Wouters RM, van Nieuwenhoven CA Hovius SER. Percutaneous aponeurotomy and lipofilling versus limited fasciectomy for Dupuytren's contracture: 5-year results from a randomized clinical trial. Plast Reconstr Surg 2018;142 (06):1523-1531

34 U.S. Department of Health and Human Services, Food and Drug Administration, Center for Devices and Radiologic Health. Summary of Safety and Effectiveness Data: Radiesse. Available at: http://wayback.archive-it.org/7993/20170111141153/http://
www.accessdata.fda.gov/scripts/cdrh/cfdocs/cfTopic/pma/pma. cfm?num=p050052s049. Accessed July 2019

35 U.S. Department of Health and Human Services, Food and Drug Administration, Center for Devices and Radiologic Health. Summary of Safety and Effectiveness Data: Restylane ${ }^{\circledR}$ Lyft with Lidocaine. Available at: https://www.accessdata.fda.gov/cdrh_docs/pdf4/ P040024S099B.pdf. Accessed July 2019

36 Khosravani N, Weber L, Patel R, Patel A. The 5-step filler hand rejuvenation: filling with hyaluronic acid. Plast Reconstr Surg Glob Open 2019;7(01):e2073

37 U.S. Department of Health and Human Services, Food and Drug Administration, Center for Devices and Radiologic Health. Summary of Safety and Effectiveness Data: Sculptra Aesthetic. Available at: https://www.accessdata.fda.gov/cdrh_docs/pdf3/P030050S002B. pdf. Accessed July 2019

38 Redaelli A. Cosmetic use of polylactic acid for hand rejuvenation: report on 27 patients. J Cosmet Dermatol 2006;5(03):233-238

39 Lefebvre-Vilardebo M, Trevidic P, Moradi A, Busso M, Sutton AB, Bucay VW. Hand: clinical anatomy and regional approaches with injectable fillers. Plast Reconstr Surg 2015;136(5, Suppl):258S-275S

40 Jabbar A, Arruda S, Sadick N. Off face usage of poly-L-lactic acid for body rejuvenation. J Drugs Dermatol 2017;16(05):489-494

41 Teimourian B, Adham MN. Rejuvenation of the hand: fat injection combined with TCA peel. Aesthet Surg J 2000;20(01):70-71 